\title{
Los trabajos bibliográficos y la formación intelectual
}

\author{
Martha Alicia Añorve Guillén \\ Investigadora del Centro Universitario de In- \\ ves ti ga ciones Biblio te co ló gi ca de la Uni versi \\ dad Na cional Au tó no ma de Mé xi co, Mé xi co.
}

\begin{abstract}
RESUMEN
Seproponelaelaboración de biblio grafíases pecializadas, selectivas y críticasc omo un me dio in troduc to rio, sis te máti co y for ma ti vo bá si co en el do mi nio de la li te ra tu ray, por tan to, del pen sa mien to, de los avan ces, de las ten den cias, de los de sa rrollos, etc. con cer nien tes al cam po dis cipli nar en que el su je to de see for mar se. Coad yu van do, con ello tam bién, a la for ma ción de bi blió gra fosespecializados en cual quier dis ci pli na, par ti cu lar men te como una op ción den tro de la for ma ción de bi blio te có$\log$ os.
\end{abstract}

\section{ABSTRACT}

$\boldsymbol{T}$ his study proposes the ela bo ra tion of spe cia li zed, spe ci fic, and cri tical bi blio gra phies as an in troductory, systematic, and basic formative means for the mastery of literature, and therefore, of thought, ad van ces, cu rrents, de ve lopments, etc. re gar ding the field of study in which the sub ject desi res to be trained. At the same time, it pro po ses the tra i ning of bi blio graphers spe ciali zed in any discipline, especially as an op tion within the tra ining of libra rians.

\section{INTRODUCCION}

$\mathrm{E}_{\mathrm{r}}^{\mathrm{n}}$ el pre sen te ar tícu lo se pro po ne la ela boración de bibliografías especializadas como un me dio para la for ma ción in te lec tual de los que se in tro du cen en un cam po dis ci plinar, en la me di da en que este tipo de tra ba jos exige:

1) el con tac to con la pro duc ción do cu mentalde un de termi na do asun to y, por tan to, permite elconocimien to deesecampo,y

2) método; aplicación de criterios uniformes para el asien to de las re fe ren cias; obli ga a clasificar el conocimiento - demandando cri te rio y ma nifes ta ción unívoca-, lo cual per mi te ir ad qui rien do la for ma ción necesaria para reco nocer las ideas claves y su manifestación sintética.

Por otro lado, en el te rre no de la for ma ción biblio te co ló gi ca, se con tem pla la ela bo ra ción de bibliografías en el sentido expuesto y como una forma de despertar el gusto e interés por este tipo de trabajos, buscando garantizar la formación de bibliógrafos; actividad que, no obstante se revalorara en el contexto y en el movimiento documentalista de fines del siglo $\mathrm{X} 1 \mathrm{X}$, pa re ce haber per di do su sen ti do en la formación del bibliotecólogodenues tros días.
En el con tex to de la ela bo ra ción de bi blio grafías, larecuperaciónau to matizadadereferencias es de gran ayuda, no obstante el trabajo querepresentalaelaboración debibliografías especializadas selectivas y críticas requiere discernimiento, criterio y juicio humano.

Así, la au to ma ti za ción y el aná li sis bi blio métrico, he rra mien tas que se pre sen tan como auxiliares valiosos en el trabajo bibliográfico, no sustituyen ni son suficientes para la compren sión y la valo ra ción del co no ci mien to, refle ja do en los re gis tros del co no ci mien to y de lain for mación. El trabajoy elen riquecimiento hu ma no que se po si bi li ta por el ma ne jo inte lec tual de los do cu men tos es algo que debe res ca tar se en el jus to va lor que tie ne en la formaciónintelectual.

En el me dio bi blio te co ló gi co se sos tie ne que, fren te a los be ne fi cia rios de los pro duc tos bibliográficos, éstos son una fuente de conocimien to, de di fu sión de los re gis tros del co nocimiento y de la información, además de una posibilidad de ordenamiento y control de la produc ción, en un mun do de ex plo sión do cumen tal que be ne ficia o está di ri gi da a loses tudiosos de la produc ción universal, na cionalo de una materiadeterminada. Así, los produc- tos bibliográficos se plantean como un auxiliar importante para que "otros" arriben a las fuentes del saber.

En con se cuen cia, se sos tie ne que quie nes elaboren esos trabajos de ben conocer, manejary apli car la in vestiga ción do cu men tal: su mé todo y sus téc ni cas (téc ni cas bi blio grá fi cas normalizadas), así como conocer el comportamiento y las fuentes del campo que se pretenden trabajar, asunto que exige, por principio, introducirse al tema y a su bibliografía.

Por otra par te, es cla ro que todo pro fe sio nis ta debe for mar se en su cam po y tie ne que co nocer la literatura correspondiente para valerse de ella en su vida estudiantil y profesional. Muchos de ellos escribirán y, sin embargo, ¿cómo hacerlo sin documentarse, sin saber cómo asentar una ficha bibliográfica, una cita; sin ha ber se for ma do en la prác ti ca del resu men y de la va lo ra ción de au to res, de en foques so bre un asun to? ¿C ómo ha cer lo si en la es cue la no se les dio la opor tu ni dad for mal de familiarizarsecon la litera tu ra de la disciplina que eligieron?

Partiendo de la formación que debe alcanzar un biblio te cólogoen elám bi to bibliográfico, 
¿cómo ini ciar la y per mi tir que se con so li de si no es otor gán do le la im por tan cia que su prácticamerece den tro del progra made es tu dios?

La in ten ción del pre sen te es cri to es, en suma, hacer ex plíci tacier ta com pren sión del trabajo bibliográfico como una fuente de formación intelectual, que seguramente es clara para mu chas per so nas, pero que no ha sido abor dadaen la li te ra tu rabiblio te co ló gi caen el sen tido aquí pro pues to.

\section{DE LOS PRODUCTOS DEL TRABAJO BIBLIOG RAFICO}

Los pro duc tos del tra ba jo bi blio grá fi co se divi den en dos gran des gru pos: las bi blio gra fía de carácter general y las especializadas. Como gé ne ro, la pri me ra se pro po ne dar noticia de todo tipo de re gis tros del co no ci mien to y de la in for ma ción, sinimpor tar el tema de que tra ten, abar can do a su vez la pro duc ción internacional o nacional. La segunda se centra en la pro duc ción so bre un tema, materia, enfoque, autor, personaolugar, enámbitos geográficos que van desde lo internacional has talolocal.

Lasbibliografías (generalesoespecializadas) pueden ser a su vez exhaustivas o selectivas por el uni ver so de pro duc ción que abar quen; dentro de ello pueden abocarse a la producción de una época pasada (retrospectivas) o referir la producción actual y aquella que se está produciendo.

La forma en que las bibliografías dan noticia de una producción determinada es a través de la presentación de fichas o referencias de las obras que la conforman. Estas fichas pueden referirúnicamente la descripciónfísica de las obras (asun to con el cual es ta rían lle nan do mínimamente su cometido) o agregar a la referen cia un re su men des crip ti vo, ana lí ti co o crí tico.

Por el tipo de asiento, o posibilidades de conocimiento de las obras incluidas en las bibliografías, éstas pueden ser descriptivas, analíticas (Milla res Car lo, 193-194, Es ca mir la, h. 8-9, Mar tí nez de Sou sa p. 66) ${ }^{1}$ y críticas (Escamilla, h. 9, Martínez de Sousa, p. 67). ${ }^{2}$ Ro drí guez Ga llar do (h. 10) apun ta que las bibliografías ge ne ra les han ten di do a la des crip- ción. Lo que se es pe ra de las bi blio gra fías espe cia li za das es que re duz can la en tro pía de la información producida en un campo, seleccionando lo valioso; de tal suerte que existe una ten den cia a que és tas sean se lec ti vas con base en los ob je ti vos, tipo de pú bli co a que se destinan y a la calidad e importancia de los contenidos, y sean críticas en el sentido en que Escamilla y Martínez de Sousa lo señalan.

Las bibliografías especializadas selectivas y críti cas son las que ma yo res ser vi cios pres tan al investigador en un campo determinado (Escamilla, h.130), más concretamente las que me jor pue den orien tar a los es tu dian tes, y son las que ma yor opor tu ni dad de for ma ción in te lec tual ofre cen a quie nes las ela bo ran.

\section{LAS BIBLIOGRAFIAS Y LA INVESTI- GACION, Y LA FORMACION INTE- LECTUAL}

En el ámbito bibliotecológico, los productos biblio grá fi cos no son con si de ra dos como traba jo de in ves ti ga ción. Se les aso cia y re du ce a su par te téc nicay, en el me jor de los ca sos, se les ve como un componente del control bibliográfico, para no perder noticiae iden tifica ción (secon si de ra que pro por cio nada tos y, a lo sumo, información) de la producción en un determinadocampo o materia y de lo que se ha ge ne ra do en un de ter mi na do perío do, en un país o en el mundoentero.

No obstante, la bibliografía, en tanto instrumento para ter ce ros que la usa rán, se re co noce como un me dio para la di fu sión, el co no cimiento y valoración de documentos. Se reconoce también que su elaboración exige investigación y conocimiento de los materiales, de la producción, del vocabulario propio del campo, de las tendencias y escuelas de pensamiento de que trata la bibliografía.

Dejan do de ladoel proble ma que pu die raconsi de rar se como más téc ni co den tro del tra bajo bibliográfico (la transcripción, descripción y aun el de la clasificación), seplan teaalgu nas condiciones estrechamente relacionadas con la formación de quienes la abordan: capacidad y gus to por la in ves ti ga ción, co noci miento de la literatura y capacidad de análisis, de valoración y síntesis. Para lograr como meta inmediata tales cualidades en quienes elaboran bibliografías, se propone concretamente a las bi bliografías es pe cia li za das, se lec ti vas y crí ti cas como un me dio para esa for ma ción, y para la formación intelectual.

Se contempla, pues, a este tipo de bibliografías como meta y como un re fle jo de la con solidación en el conocimiento de una temática determinada. Con tal intencionalidad, y tratándolo como un problema de enseñanza-aprendizaje, su tratamiento debe atender a la gradación del co noci mien to, acor de conalgut nos principio de enseñanza-aprendizaje. Se recomienda que la formación intelectual por me dio del mane jo de docu men tos, y con cre ta men te la for ma ción bi blio grá fi ca, se en ca mine de lo sim ple a lo com ple jo, de lo co no ci do a lo des co no ci do y de lo con cre to a lo abs tracto, empezando por literatura introductoria y con referencias descriptivas a lo sumo, con resúmenesdescriptivos, buscándodesarrollar la ca pa ci dad de análi sis y de sín te sis.

Mediante el conocimiento de la literatura se puede desarrollar el pensamiento independiente, analítico, crítico y fundamentado; en suma, la for ma ción de un cri te rio nue vo y propio que resulta necesario para unaverdadera con tri bu ción al de sa rro llo y re no va ción de las disciplinas.

Los proce sos y lo gros al can za dos por el traba jocon la li te ratura en ri que cen y mo di fican no sólo la parte intelectual de los individuos, sino que contribuyen a formary for ta le cer la personalidad en su conjunto.

En el en ten di do de que “...en la for ma ción uno se apro pia por en te ro aque llo en lo cual y a través de lo cual uno se for ma." (Ga da mer, p. 40) y de que "For man do al ob je to, ... la con cien cia que trabaja se ele va por en ci ma de la in me diatez de su es tar ahí a la ge ne ra li dad; ... y asíformando a la cosa se for ma a sí mis ma..." (Gadamer, p. 41); en ese contexto se propone al trabajobibliográ fi co como un me dio para que los alum nos se for men en el co no ci mien to, en la com pren sión, en la apro pia ción in te lec tual de sus cam pos dis ci plina res y como for ma de que la disciplina cobre para ellos un sentido integral y vívido.

1 En Agustín Millares Carlo, las bibliografías descriptivas tienen el propósito de “...dar a conocer con exactitud la condición material de los libros, es decir sus caracteres externos, como el nombre y apellidos de sus autores, título exacto, fecha y lugar de publicación, nombre del impresor, editor, tamaño, número de página, particularidades tipográficas, ilustraciones, a veces el precio, y, para los libros antiguos, otros detalles como calidad del papel, procedencia, encuadernación, etc." En tanto que las analíticas, además de lo anterior, pretenden dar a conocer el contenido, su preocupación es el texto mismo. (El subrayado no es de Millares.) Tanto Escamilla como Martínez de Sousa señalan para las bibliografías descriptivas la misma pretensión y características, no así para las bibliografías analíticas. Para Escamilla la ficha de estas últimas (que denomina también como bibliografías anotadas) va acompañada de un análisis o de un resumen. "Lleva anotaciones que son muy variadas: transliteraciones o traducciones de títulos en alfabetos o idiomas extranjeros; interpretación de títulos cuyo significado no es evidente; información sobre aspectos materiales o históricos de las obras."

2 En Martínez de Sousa encontramos que las bibliografías analíticas añaden a la referencia un juicio crítico o un análisis. No obstante define lo que es una bibliografía crítica, a la que menciona como sinónimo de bibliografía comentada. Para este último autor, la ficha en bibliografía crítica va acompañada de un juicio crítico u orientador de su contenido, validez y contribución. Es este el sentido en que Escamilla caracteriza a la bibliografía crítica, señalando que ésta exige el examen del texto de la obra, para expresar un juicio crítico sobre la misma, que permita conocer su valor. 
Por otro lado, la lo ca li za ción y co no ci mien to de la li te ra tu ra re la cio na da con un tema dado es generalmente el primer trabajo que emprende un investigador o un estudioso. Este conocimiento permite no duplicar investigaciones, co no cer po sibles en cau ces de nue vos trabajos, conocer a autores relacionados con la ma te ria, ten den cias, etc. al gra do de po der afirmar, como Zol towki hi cie ra para los productos bibliográficos, que tanto éstos como su pro ce so y la ex plo ra ción de la li te ra tu ra de manera graduada, metódica e intencionada, pueden con du ciral des cu bri mien to de ci clos de crea ción in te lec tual o ar tís ti ca (Zol towski, Vic tor, ci ta do en Malclés p.12).

En los tratados introductorios a la investigación, cuando se plantean los diferentes tipos de ésta, se hace referencia a la investigación do cu men tal. Este tipo de in ves ti ga ción se caracteriza por tener como base el análisis de ciertos registros del conocimiento o de la infor ma ción. A este tipo de in ves ti ga ción per tenece la bibliografía.

Es ca mil la, (h. 4) adop ta la de fi ni ción de bi bliogra fía que Malclés ofre ce en Cours de bibliographieàl'inten tion desétudiants del'université et des candidats aux examens de bibliothécaires: "la bibliografía es el conocimien to de to dos los tex tos im pre sos y está basada en la investigación, transcripción, descripción y clasificación de esas obras con ob je to de ela bo rar los ins tru men tos de tra ba jo intelectual, llamados repertorios bibliográficos o bi blio grafías." En tal de fi ni ción se des ta ca el procedimiento a seguir en el trabajo bibliográfico y el producto que se obtiene. Respecto a lo primero, se aclara que el punto de par ti da del tra ba jo bi blio grá fi co es la in vestigación, mediante la cual se determinan las obras que for ma rán la bi blio gra fía, de acuer do al tema se lec cio na do, al uso y usua rio al que se dirigen. Por otra parte, la descripción de las obras re mi te no sólo a su des crip ción ex ter na o físi ca, sino tam bién a su con te ni do in te lec tual, que se da me dian te un re su men, aná li sis o comentario.(Escamilla, h. 4-5)

Bajo la idea de que las bi blio te cas y las bi bliografías no presentan manojos de obras, sino un cuerpo de materiales con relaciones entre sí, el traba jo bi blio gráfi co por ma te ria y/o por épo ca ofre ce con jun tos re la cio na dos y de rela cio nes; ofre ce, portan to, una fuen te de formación en el conocimientode una disciplina y de las ten den cias de la li te ra tu ra de una época, tanto para quien lo elabora como para quien lo utiliza.

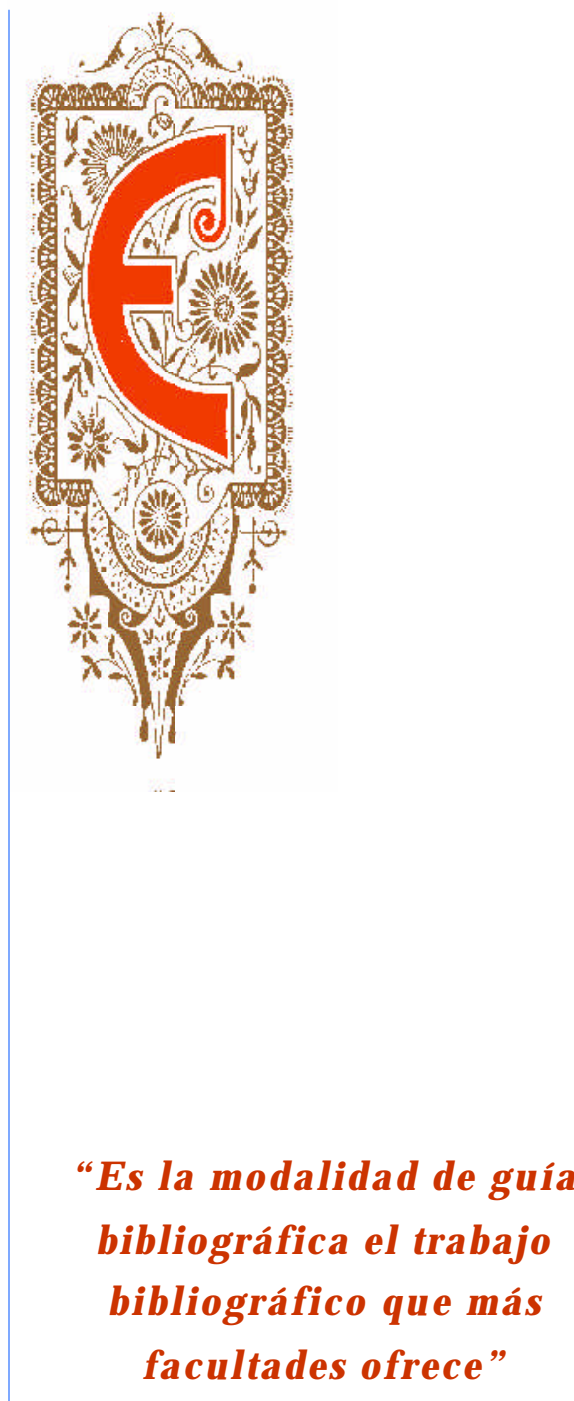

Malclés (p. 9) ve a las bi blio gra fías o re per torios bibliográficos como uno de los instrumentos del trabajo intelectual, en donde los repertorios especializados tienen como meta característica el de informar sobre la actividad intelectual registrada sobre algún tema -bien sea a ni vel na cio nal o in ter na cio nal, de manera exhaustiva o selectiva, retrospectiva o en cur so- y pu dien do pre sen tar des de una lista descriptiva de las obras, hasta acompañar las por un co men ta rio crítico.

Es la mo da li dad de guía bi blio gráficael tra bajo bi blio gráfi co que más fa cul ta des ofre ce yel que más se acerca a la po si bi li dad se ña la daen el párra fo an terior. Su ela bo ra ción exi ge fijarse un ob je ti vo, di ri gir la a un usua rio y a un uso potencial: a ta les asun tos se su bor di na la selección de las obras que la constituirán. Demanda además límites que marquen (ya no des de el pun to de vis ta del para qué, el qué y el a qué ni vel de profun di dad) fron te ras de tiempo, de idio ma, de geo grafía, que, en con jun to, arrojan luz so bre sus po si bi li da des. De tal mane ra este tipo de tra ba jos in ten ta ofre cer conjuntos significativos, conocimientos, enfoques, corrientes, hallazgos, frente a un fenómeno. Desde esa perspectiva es posible afir mar que tan to su ela bo ra ción como su uso ofrecen grandes posibilidades de formación intelectual.

\section{A MA NERA DE CON CLU SIONES}

Se propone retomar y aprovechar, formal y sistemáticamente, la posibilidad de formación intelectual que en su conjunto ofrece el trabajo de productos bibliográficos y de exploración de la literatura para una investigación o estudio. Se hacen explícitas las virtudes que este trabajo ofrece, enseñando a los alum nos que, al for mar a la cosa, ellos se forman, y que ha ber se aven tu ra do por el es tu dio de una disciplina significa formarse en algo que de otra manera no se hubiese intentado.

En la for ma ción bi blio te co ló gi ca, el efec to de re to mar esta idea im ple men tar la es do ble en la me di da en que los alum nos de esta dis ci plina, como la de cual quier otra, de ben for mar se intelectual y plenamente en su campo discipli nar y en que, por otro lado, de ben for mar se para elaborar bibliografías como parte de su traba jo en uni da des de in for ma ción y, en úl timo caso, pue dan de ci dir de di car se a la bibliografía como tra bajo es pecial. 


\section{OBRAS CONSULTADAS}

ESCAMILla GONZALEZ, Gloria. Bibliografía e investigación bibliográfica.Seminariode Investigaciones Bibliotecológica, Serie B, no. 1. México, D.F: La autora, 1960. 200 h. Tesis de Maestría en Bibliotecología. UNAM. F.F.L.

GAD AMER, Hans- Georg. Verdadyméto do: fundamentos de unahermenéutica filosófica. Hermeneia, 7. 3 ed. Salamanca, España: Ediciones Sígueme, 1988. 687 p.

MALCLES, Loui se Nóelle. Labibliografía . Trad. Ro ber to Jua rroz, revi sióntéc nica Jo se fa Sabor. Buenos Aires, Argentina:EUDEB A. Editorial Universitaria de Buenos Aires, c1960. 71p.

MARTINEZ DE SOUSA, José. Diccionario de bibliología y ciencias afines. Biblioteca del Libro. Salamanca, Madrid. España: Fundación Germán Sánchez Ruipérez, 1989. 852p.

MILLARES Carlo, Agus tín. "La bi blio gra fía y las bi blio gra fías". Cuadernos Americanos. LXXIX (enero- febrero, 1955). p.176-194.

RODRIGUEZ GAllardo, José Adolfo. Guía de materiales de referenciasobrelashumanidadesmexicanas. Aus tin, Te xas: El autor, 1970. 269 h. Tesis de Maestría en Bibliotecología. The University of Texas at Austin. Faculty of Graduate School.

ZOL TOW KI, Victor. "Les cy cles de la créa tion in te lec tuel le et ar tis tique". Année sociologique, 1952. 